\title{
Interoperability of a Mobile Health Care Solution with Electronic Healthcare Record Systems
}

\author{
P. De Toledo, W. Lalinde, F. Del Pozo , Senior Member, IEEE, D. Thurber and S. Jimenez-Fernandez
}

\begin{abstract}
Mobile health care solutions involving patient monitoring are an increasingly accepted element in chronic disease management strategies. When used in healthcare systems with different providers, it is essential that the information gathered from the patient is available at each of these providers' information repositories. This paper describes the design of a connectivity interface based on the HL7 standard that allows the MOTOHEALTH mobile health care solution to communicate with external electronic healthcare record systems supporting $\mathrm{HL} 7$.
\end{abstract}

\section{INTRODUCTION.}

$\mathrm{M}$ obile health care solutions are growing as an alternative for chronic disease management. Reported experiences exist in the field of Diabetes [1], Chronic Obstructive Pulmonary Disease [2] and Heart Disease [3][4].

It is widely accepted that finding a suitable business model for mobile telehealth is one of the current barriers for complete acceptance of these services. These business models have to address several issues, such as reimbursement for telemonitoring and other telemedicine services, security of patient information, liability, etc. Together with these, the way to re-engineer existing processes for patient care to adapt them to mobile telehealth, is a key factor for success. In the USA, with a private insurance healthcare system, most current telemonitoring services are run by organisations like HMOs (Health Maintenance Organizations) or the Veterans Health Administration, that are in charge of all aspects of patient care. In Europe, where most countries follow the Beveridge model, based on taxation and with many public providers, or the Bismarck 'mixed' model, funded by a premium financed social insurance system and with a mixture of public and private providers, it seems more difficult to introduce

Motorola Inc

This project is partially funded by

Paula de Toledo, Francisco del Pozo and Silvia Jiménez-Fernández are with the Bioengineering and Telemedicine Research Centre of the Technical University of Madrid - Spain. Paula de Toledo is now visiting professor at the Carlos III University of Madrid, while Silvia Jiménez-Fernández is now Associate Professor in University of Alcalá - Spain. (e-mail: paula@gbt.tfo.upm.es).

William Lalinde and Douglas Thurber are with Motorola Inc. (e-mail edt010@motorola.com). telemonitoring practices for chronic disease management. In these countries any successful telemonitoring experience has to be seamlessly integrated with existing health care models, both at an organisational and an informational flow level.

The four current options for arranging telemonitoring services are a) running it from inside the health care organisation [1-3]; b) outsourcing the technical and logistics part to a specialised partner that acts as a service provider and conveys the information to the health care provider; c) a mixed option where a logistics partner does exist but it depends organisationally on the health care organisation that provides the service [4] and d) wellness monitoring scenarios were there is no provider, but the user records vital signs for his own use, and which is outside the scope of this work.

The outsourced model offers many advantages, as most health care organisations do not have the appropriate structure and personnel to take care of the logistics (helpdesk, device replacement in case of failure, stock management) and the technical maintenance that such services demand. This outsourcing could be run by specialised companies, mobile telecom operators or by other traditional providers such as home gas therapy suppliers. A single telemonitoring provider could, in this model, assist patients from different health care providers, profiting from economy of scale.

Despite its advantages, this model has its main weakness in the integration with health care providers. Information regarding a patient should be immediately available to his/her care team, and be reflected in his Electronic Healthcare Record (EHR). This involves exchanging information with information systems from different organisations

This paper describes the design of a standards-based Connectivity Interface designed to interconnect the mobile telehealth solution MOTOHEALTH with electronic healthcare record systems from external providers, increasing the suitability of this technical solution to different business models for mobile telehealth.

\section{THE MOTOHEALTH SOLUTION}

The MOTOHEALTH solution is a telemonitoring platform designed for chronic disease management [6], 
using a Motorola cell phone.

The user terminal (Figure 1) consists of this cell phone, acting as gateway for telemonitoring information, and a set of devices, that may be used or not depending on the patient's condition: pulse oximeter, spirometer, glucometer, sphygmomanometer and scale. This collection of supported sensors makes the solution appropriate for the management of a wide range of chronic diseases such as congestive heart failure, chronic obstructive pulmonary disease and diabetes.

Communication between cell phone and sensors is wireless for greater usability and robustness, based on the Bluetooth standard.

The cell phone connects to the MOTOHEALTH Application Server (Figure 2) using 3G or iDEN mobile data packet communications. Data are stored and made available to the patient's care team through a web-based application. The MOTOHEALTH application server supports the generation of notifications if results received are outside normal ranges.

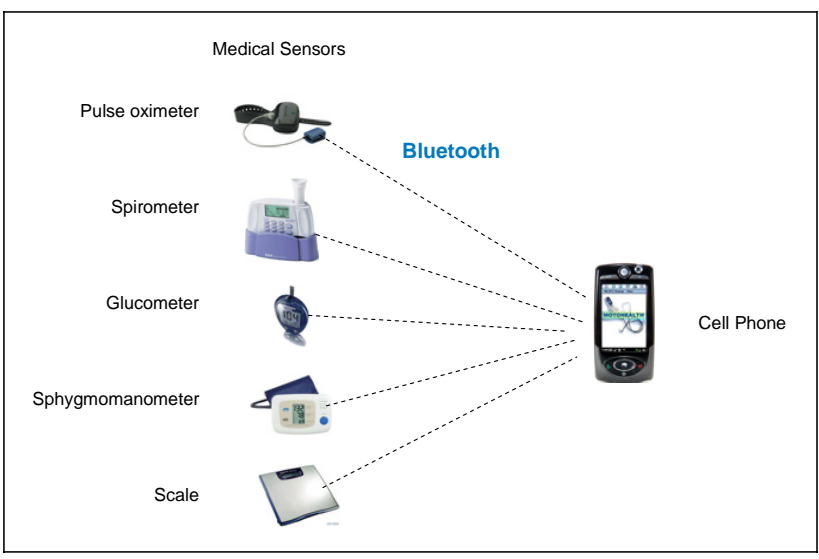

Figure 1. MOTOHEALTH User Terminal.

MOTOHEALTH solution is currently being used in a set of field evaluations in the USA, UK and Spain.

This paper describes the MOTOHEALTH Connectivity Interface, an interface designed to communicate the information received at MOTOHEALTH with the greatest possible number of EHR and other patient information databases, thus making possible the use of MOTOHEALTH in healthcare systems with many providers involved in patient care.

\section{USE OF STANDARDS}

This interoperability goal leads us to the use of standards. Main standards in the EHR field are EN 13606 [7] and HL7 [8], which are starting to converge in some aspects. The main difference among them is that EN 13606 standardizes the generic architecture of a communicable healthcare record, while HL7 is limited to the definition of standard messages for the exchange of the information that is more frequently transferred among systems. The latest version of HL7, 3.0, proposes a Clinical Document Architecture, which is closer to a full electronic record architecture.

Although EN 13606 is a more powerful standard it was not considered appropriate for our project, mainly due to 1) a complete stable version is not available yet and 2) it has not yet been adopted by any commercial solution.

We selected HL7 version 2.3 due to 1) its widespread adoption by the industry and 2) its supports of all the integration needs identified in MOTOHEALTH. HL7 is used in most tertiary hospitals in Europe to interface different information systems (mainly EHR and laboratory systems). Some national and regional initiatives plan to use it in the near future as the basis of an integrated regionallevel EHR.

\section{REQUIREMENTS}

The team involved in MOTOHEALTH in Spain had already a long experience in chronic patient telemonitoring, mainly with respiratory patients [9]. This experience, together with a literature review was used to specify the information that had to be exchanged with the EHR system. This information is summarized in Table 1 . When a patient is admitted into a chronic disease management program supported by MOTOHEALTH, his/her personal information will be usually already available at an Admission/Discharge/Transfer (ADT) system or Electronic Healthcare Record system. To avoid the repetition of data introduction into MOTOHEALTH, it is desirable that the admission of a patient be automated, by means of the exchange of a message.

Once the patient is admitted, he/she will perform a daily monitoring session and this information will be sent to MOTOHEALTH. In some service models, this information will have to be sent immediately to the patient's provider information system, while in others, main follow up tasks will be done by the telemonitoring service provider, and only periodic summarized reports will have to be sent, i.e. to his general practitioner or to the hospital for recording it at the EHR.

TABLE.1. INFORMATION EXCHANGE REQUIREMENTS

\begin{tabular}{ll}
\hline \hline $\begin{array}{l}\text { From } \\
\begin{array}{l}\text { Telemonitoring } \\
\text { system to EHR }\end{array}\end{array}$ & $\begin{array}{l}\text { - Send results from a single telemonitoring session } \\
\text { over one period }\end{array}$ \\
\hline $\begin{array}{l}\text { From EHR to } \\
\text { Telemonitoring } \\
\text { system }\end{array}$ & - Register a patient in telemonitoring system \\
\hline \hline
\end{tabular}

It was also considered whether to send these updates each time a result was received (unsolicited update) or only when the EHR system requests it (queries) [10]. The unsolicited update mode is nowadays supported by more EHR systems, and therefore it was implemented first. 


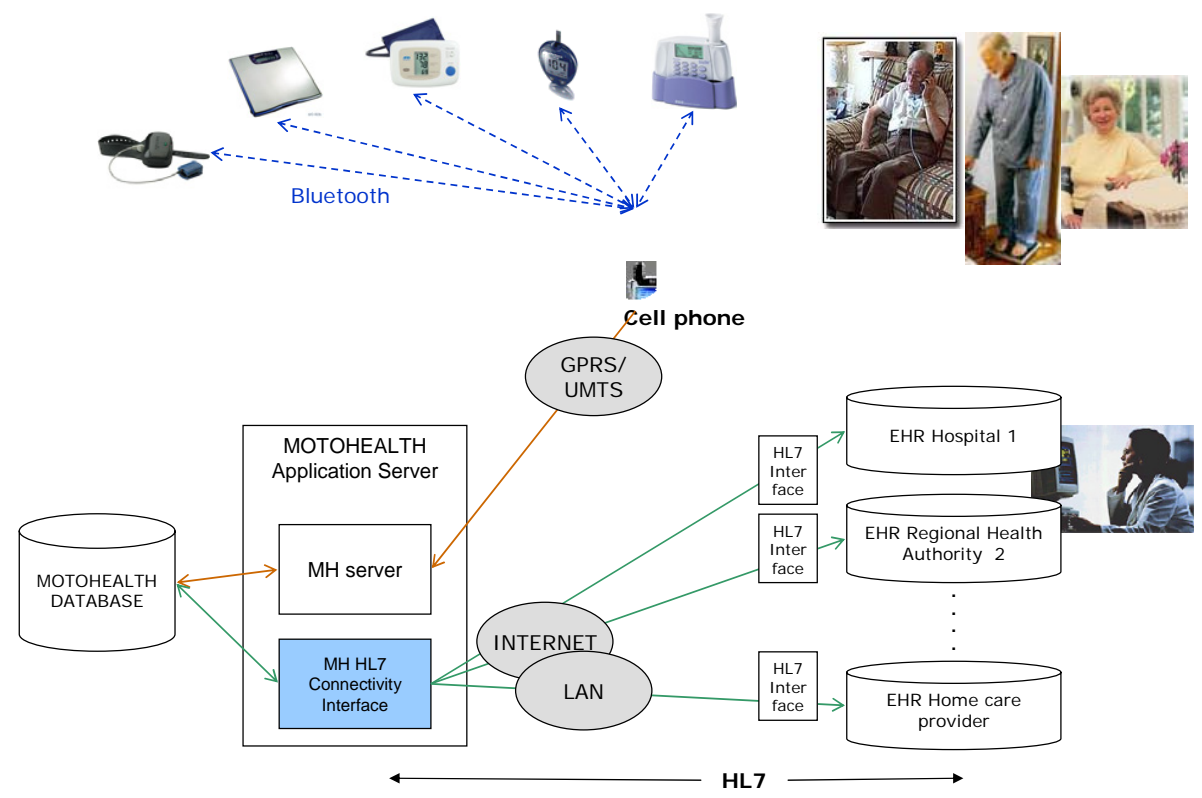

Figure 2. MOTOHEALTH Connectivity Interface

\section{SPECIFICATION AND DESIGN}

\section{A. HL7 Message for telemonitoring results}

The message selected for sending telemonitoring results to the EHR system, is ORU^RO1 Unsolicited transmission of an observation message. This message implements the transmission of any kind of clinical observations including clinical laboratory results, ECG, pulmonary function studies, measures of patient status and condition and vital signs.

In the case of MOTOHEALTH, ORU^RO1 observation messages are not initiated by an order message as is the case in traditional laboratory systems. Using this unsolicited mode, the producing service returns the value of an observation as soon as it is available.

One result segment (OBX) is transmitted for each observation component (such as blood pressure or glucose levels). A sample message is shown in Table 2.

TABle. 2. SAMPle HL7 MESSAgE

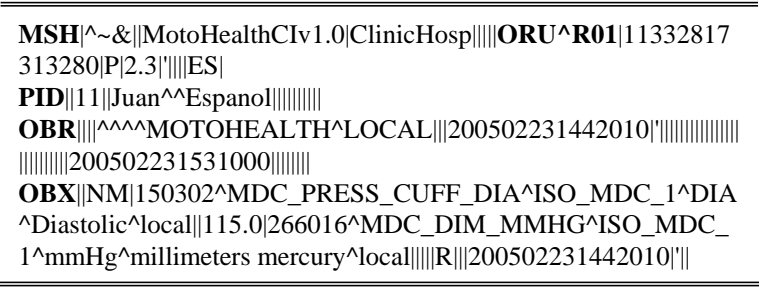

\section{Message acknowledgement}

HL7 standard supports two different message acknowledgment modes: original mode and enhanced mode.
Original mode involves acknowledging a message when it is committed to safe storage, and is the one implemented by the MOTOHEALTH Connectivity Interface. If observation messages are not acknowledged after a configurable time, they are sent again.

\section{Coding systems for observation reporting}

To report results of telemonitoring sessions, there is a need to code the tests performed in a way an external system is able to understand. Coding systems considered are described in Table 3.

TABLE. 3. CODING SYSTEMS FOR OBSERVATIONS.

\begin{tabular}{|c|c|}
\hline $\begin{array}{l}\text { ISO / IEEE } \\
1073 \text { Part } \\
10101 \\
\text { Nomenclature }\end{array}$ & $\begin{array}{l}\text { The Point of Care Medical Device communication } \\
\text { (PoCMDC or 1073) is a family of standards approved by } \\
\text { both IEEE and CEN, that contains, among other } \\
\text { specifications, a nomenclature for Point of Care medical } \\
\text { devices and tests. }\end{array}$ \\
\hline $\begin{array}{l}\text { LOINC® } \\
\text { (Logical } \\
\text { Observations } \\
\text { Identifiers, } \\
\text { Names, } \\
\text { Codes), }\end{array}$ & $\begin{array}{l}\text { Clinical terminology for laboratory test orders and } \\
\text { results, produced by the Regenstrief Institute, providing } \\
\text { a set of universal names and ID codes for identifying lab } \\
\text { and clinical tests. Identified by HL } 7 \text { in } 1999 \text { as a } \\
\text { preferred code set for laboratory test names.. }\end{array}$ \\
\hline $\begin{array}{l}\text { Clinical Data } \\
\text { Interchange } \\
\text { Standards } \\
\text { Consortium } \\
\text { (CDISC). }\end{array}$ & $\begin{array}{l}\text { Non-profit organization committed to the development } \\
\text { of industry standards to support the electronic } \\
\text { acquisition, exchange, and archiving of clinical trials } \\
\text { data and metadata. }\end{array}$ \\
\hline
\end{tabular}

Both ISO / IEEE Point of care medical device communication [11] ( "ISO_MDC_1" in HL7 messages) and LOINC [12] ("LN" in HL7) are well suited to our needs, as most of the tests performed at the homes by MOTOHEALTH devices can be coded with both these 
nomenclatures. PoCMDC is best suited to our goals from a conceptual approach, as it is specific to point of care monitoring, and it can be considered preferable for this reason, while LOINC is widely used by systems accepting information from Laboratory systems, which is an advantage. Selection of a coding scheme can be configured on a per installation basis.

\section{Coding systems for units}

Units of the observation values do also need to be coded in a standard system. ISO single case units and ISO derived units codes have been used when existing. ISO/IEEE 1073 codes have been used elsewhere. MOTOHEALTH internal codes are provided as well.

\section{B. HL7 Message for registering patients}

This message is used to avoid the manual introduction of patient data when they are registered in the telemonitoring service. This is particularly useful for initial loads of patients into MOTOHEALTH. ADT^A28 message has been selected, as it is not related to the notification of real-time ADT events (as are all other Admission Discharge and Transfer HL7 messages, like ADT^A04 -register a patient or $\mathrm{ADT}^{\wedge} \mathrm{A} 05$-pre-admit a patient) and does not require exchanging information regarding patient visits.

\section{System Design}

HL7 is a standard addressing only the application layer (7th layer) of the ISO/OSI network model, and therefore it can be implemented over different transport and communications protocols. For this implementation we have used TCP/IP in the lower layers.

The security of the communication is an essential requirement for transmitting patient related information. Messages transmitted contain patient identifiable data (name) together with health information (observation results) and therefore have to be encrypted before transmission. MOTOHEALTH Connectivity Interface implements the Secure Sockets Layer protocol for secure transmission of data.

\section{CONCLUSION}

The Connectivity Interface will be tested in the MOTOHEALTH field evaluation taking place at Clinic Hospital at Barcelona. This test will show if the level of integration achieved is adequate for the needs of chronic patient management in a healthcare system like the Spanish one, with many providers, and determine if extended functionality like the use of queries and the generation of periodic summarised reports is of interest. We also expect to get further insight on the impact of information systems integration in the service and business model for mobile healthcare solutions.

From the technical point of view, future work involves considering if a new release of the system based on web services technology and using XML-formatted HL7 messages can improve the performance for the future and expand the number of compatible systems.

More specialised functionality such as allowing the healthcare professional to directly modify the periodicity and type of tests and send this configuration to the cell phone (instead of delegating these tasks in the telemedicine service provider) is not demanded at this point, but may be of interest in the future, when professionals and patients become more aware of the capabilities of Mobile Health Care Management.

\section{ACKNOWLEDGMENT}

The authors want to thank Dr. Roca's team in Clinic Hospital at Barcelona, Spain for their contribution to the specifications of the system, as well as Raquel Gonzalez Couto, Laura Alonso Nevado and Nestor Sanchez Perez for the development work.

\section{REFERENCES}

[1] E. Hernando. EJ Gomez, García-Olaya A, Torralba V. Del Pozo F. A Mobile Telemedicine Workspace for Diabetes Management.MHealth: Emerging Mobile Health Systems. Series International Topics in Biomedical Engineering. R. Istepanian, S. Laxminarayan and C. Pattichis (editors), Kluwer Academic/Plenum Publishers, Ed. Springer, pp587-600.

[2] Del Pozo F, de Toledo P, Jiménez S, Hernando E, Gómez EJ. Chronic Patient's Management: the Copd Example.M- Health: Emerging Mobile Health Systems, R. Istepanian, S. Laxminarayan and C. Pattichis (editors), Kluwer Academic/Plenum Publishers, pp 575-586.

[3] Schofield RS, Kline SE, Schmalfuss CM, et al Early outcomes of a care coordination-enhanced telehome care program for elderly veterans with chronic heart failure. Telemed J E Health. 2005 Feb;11(1):20-7.

[4] C.H. Salvador, M.P. Carrasco, M.A. de Mingo et al. "Airmed-cardio: a GSM and Internet services-based system for out-of-hospital follow-up of cardiac patients”. IEEE Trans Inf Technol Biomed. 2005 Mar;9(1):73-85.

[5] Lameire N, Joffe P, Wiedemann M. Healthcare systems--an international review: an overview. Nephrol Dial Transplant. 1999 Dec;14 Suppl 6:3-9.

[6] Istepanian R, Lacal J, Prag N. Proceedings of the Telemed and eHealth 2005 conference pp 65. To be published in Journal of Telemedicine and Telecare.

[7] ENV13606 - CEN/TC251. Electronic Healthcare Record Communication. Parts 1, 2, 3 and 4. Pre-standard. http://www.centc251.org/.

[8] HL7 standard. www.hl7.org. Available on line

[9] De Toledo P, Jimenez S, Del Pozo F., Roca J, Alonso A, Hernandez C., "A telemedicine experience for chronic care in COPD", IEEE Transactions on Information technology in biomedicine. To be published July 2006.

[10] Spronk R. HL7 Query messages. Ringholm White Papers. Available Online http://www.ringholm.de/docs/00300_en.htm.

[11] IEEE Medical Device Communications / Health Informatics: Point Of Care Medical Device Communications standard. Part 1. Nomenclature. Available online http://www.ieee1073.org. 